FACTA UNIVERSITATIS

Series: Mechanical Engineering Vol. 17, N N $^{\mathrm{o}}$, 2019, pp. 47 - 64

https://doi.org/10.22190/FUME190121010H

Original scientific paper

\title{
A STUDY ON GROSS SLIP AND FRETTING WEAR OF CONTACTS INVOLVING A POWER-LAW GRADED ELASTIC HALF-SPACE
}

\author{
Markus Heß \\ Department of System Dynamics and Friction Physics, Berlin University of Technology, \\ Germany
}

\begin{abstract}
For the steady wear state of two contact problems involving power-law graded materials, closed-form solutions are derived in terms of pressure distribution and limiting shapes of profile. Both gross slip of an initially flat-ended cylindrical punch on a powerlaw graded half-space and the load-controlled fretting wear under partial slip of an initially parabolic indenter are studied. In the case of gross slip at fixed penetration depth there exists a certain exponent of elastic inhomogeneity, for which the effective volume change takes its maximum value. To minimize wear due to fretting under partial slip, an amplitude dependent design of the material gradient is necessary. For large amplitudes of the tangential force a gradient ranged from a soft surface to a hard ground is beneficial, small amplitudes require a reverse gradient characterized by a hard surface and a soft ground. However, the choice of the material gradient also has a decisive influence on the strength of stress singularities at the contact edge and thus the initiation of fretting fatigue cracks, which is why it is discussed in more detail.
\end{abstract}

Key Words: Contact Mechanics, Gross Slip, Partial Slip, Fretting, Wear, Functionally Graded Material

\section{INTRODUCTION}

If the normal contact of two bodies is superimposed by small tangential oscillations, partial slip occurs within the contact interface which causes wear. This phenomenon is called fretting and arises in numerous engineering applications like power steam generator

\footnotetext{
Received January 21, 2019 / Accepted March 15, 2019

Corresponding author: Markus $\mathrm{He} ß$

Department of System Dynamics and Friction Physics, Berlin University of Technology, Sekr. C8-4, Straße des 17. Juni 135, 10623 Berlin, Germany

E-mail: markus.hess@tu-berlin .de
} 
tubes [1], roots of compressor and turbine blades [2], artificial hip joints [3], electrical contacts [4], railway axles [5], etc. Extensive investigations have been made in order to tackle the fretting problem, which can be responsible for a shortened life cycle and the failure of machine components. Most analytical considerations apply to elastically similar materials and are mainly restricted to the solution of the steady contact state, at which all partial slip is ceased and thus no further wear takes place [6, 7]. Particular attention was paid to the calculation of the limiting shape of the profiles, which does not depend on the type of wear criterion [8,9]. It merely requires knowledge of the solutions of normal contact problems between the rigid cylindrical indenters with appropriately shaped tips and the elastic half space. Due to the fact that the (minimum) stick area does not change during the transition from the initial to the steady state, the diameter of the indenter is a priori known [10]. However, the calculation of the stress evolution and profile change during the transient wear process, require an explicit wear law. Most numerical wear simulations are based on Archard's well-known wear law [11]. It has been implemented in finite element methods as well as other efficient numerical methods based on an integral equation approach or quadratic programming [12-14]. An extremely efficient and therefore very fast method for simulating fretting wear is the method of dimensionality reduction [15]. For simulations of gross slip wear it was shown that the calculation time using this method is for several orders lower than that of models based on the finite element method [16]. The method was not only used for the numerical study of classic fretting problems $[17,18]$, but also for the calculation of the limiting shape of the worn profile under multi-mode fretting and the results are in good agreement with experimental ones [19].

All the above mentioned contributions to the study of fretting wear have in common that they only consider elastically homogeneous materials. Corresponding studies of elastically inhomogeneous materials are scarce although it is well-known that a controlled gradient of the elastic modulus can lead to a greater resistance to contact and friction damage [20]. For instance, Hertzian cone cracks are suppressed due to the reduction of the maximum tensile stresses in the surface [21]. One reason for the lack of research into fretting of so-called functionally graded materials lies in the difficulty of solving such problems. The few works are mostly related to special material gradients and plane contact problems, which are solved numerically $[22,23]$. A powerful numerical method for solving three-dimensional fretting contact problems involving arbitrary material gradients goes back to Wang et al. [24], however, studies on wear are not included.

In this paper we derive analytical solutions for the steady wear state of two frictional contact problems involving power-law graded materials, whose gradient perpendicular to the half-space surface is prescribed by

$$
E(z)=E_{0}\left(\frac{z}{z_{0}}\right)^{k} \text { with }-1<k<1 .
$$

Although this gradient is physically unrealistic due to a vanishing or infinitely large elastic modulus at the surface, it reflects qualitative behavior of the real gradient materials quite well. The great advantage of a material gradient according to Eq. (1) is that it is probably the only one for which contact problems can be solved in a closed analytic form. Whereas complete solutions of frictionless normal contact problems with and without adhesion have been known for some time [25, 26], solutions of tangential contacts were 
only recently developed [27, 28]. Taking into account the latter results, in Chapt. 2 we study the gross slip of an initially flat-ended cylindrical punch on a power-law graded half-space. Chapt. 3 deals with fretting wear under load-controlled conditions in the partial slip regime for the contact between a rigid initially parabolic indenter and a powerlaw graded half-space. For both problems, complete solutions are given in terms of stresses and limiting shapes of the profile. In addition, the influence of the exponent of elastic inhomogeneity as well as the tangential force amplitude on the wear volume is discussed.

\section{Gross SLIP WeAr ANALYSIS OF A RIGID PUNCH ON A POWER-LAW GRAdED HALF-SPACE}

We first consider the contact between a rigid flat-ended cylindrical punch with a circular base and a power-law graded elastic half-space as shown in Fig. 1. The rigid indenter is pressed into the half-space by an initial normal force $F_{N, 0}$, giving rise to a prescribed indentation depth $d$. Subsequently, the power-law graded half-space is moved horizontally with constant speed causing wear on the punch. In contrast, wear of the halfspace is assumed to be negligibly small. The indentation depth is kept constant during sliding. From the practical point of view, this is a hardly controllable condition. However, we later explain this choice and discuss the gross slip under a fixed normal force.

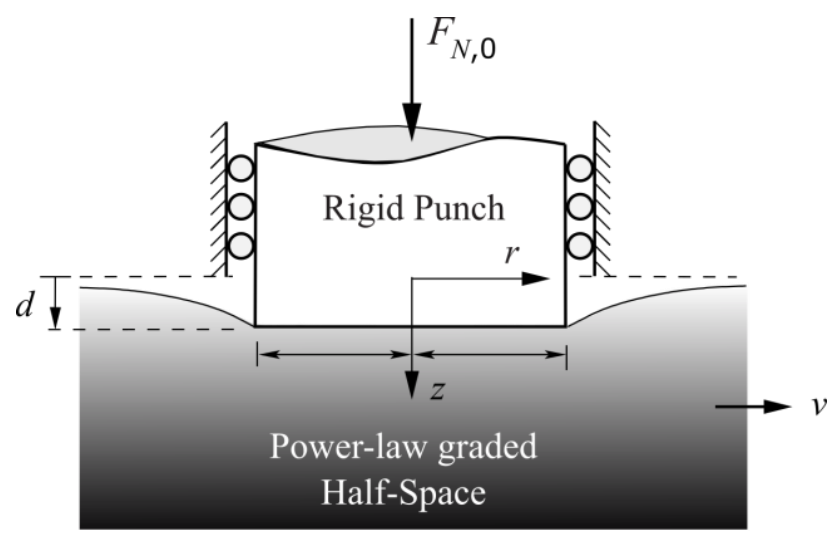

Fig. 1 Initial state of a sliding contact between a rigid flat punch and a power-law graded elastic half-space

It is supposed that normal and tangential contacts are uncoupled. Since the indenter is rigid, uncoupling is, strictly speaking, only valid for a Poisson ratio of $v=1 /(2+k)$, known as Holl ratio. Thus, negative exponents of elastic inhomogeneity would lead to unphysical values. However, more detailed studies show that there is only a very weak coupling if we consider Poisson's ratios of 0.5 instead and all solutions deviate only slightly from the solutions using Holl's ratio [27]. For this reason, we adopt it for negative exponents as well. The solution of the initial contact state of Fig. 1 can be taken from Booker et al. [25]. The normalized pressure distribution is given by 


$$
\frac{p(r) \pi a}{2 E_{0} d}=\frac{h_{N}(k, v)}{2\left(1-v^{2}\right) z_{0}^{k}\left(a^{2}-r^{2}\right)^{\frac{1-k}{2}}}
$$

where $h_{N}(k, v)$ is defined in the appendix. The pressure has a singularity at the edge of contact which becomes milder with increasing exponent of elastic inhomogeneity. The displacement of the half-space surface reads

$$
\frac{w_{0}(r)}{d}=\left\{\begin{array}{ll}
1 & \text { for } 0 \leq r \leq a \\
\frac{\cos \left(\frac{k \pi}{2}\right)}{\pi} \frac{2}{1+k}\left(\frac{a}{r}\right)^{1+k}{ }_{2} \mathrm{~F}_{1}\left(\frac{1+k}{2}, \frac{1+k}{2} ; \frac{3+k}{2} ; \frac{a^{2}}{r^{2}}\right) & \text { for } r>a
\end{array},\right.
$$

where ${ }_{2} F_{1}(a, b ; c ; z)$ represents the Gaussian hypergeometric series

$$
{ }_{2} F_{1}(a, b ; c ; z)=\sum_{n=0}^{\infty} \frac{(a)_{n}(b)_{n}}{(c)_{n}} \frac{z^{n}}{n !} \quad \text { with } \quad(x)_{n}:=\frac{\Gamma(x+n)}{\Gamma(x)} .
$$

According to Archard's wear law, the wear rate during sliding will be higher at larger pressures, so that wear takes place primarily at the edge of contact. After a sufficiently long time the pressure distribution becomes uniform and the rigid punch reaches a steady state profile. This final shape coincides with the surface displacement of a circular domain on a power-law graded half-space which is loaded by a constant pressure. The solution of the steady contact state can be taken again from [25]. The displacement of the half-space surface is

$$
\frac{w_{\infty}(r)}{d}=\left\{\begin{array}{ll}
{ }_{2} \mathrm{~F}_{1}\left(\frac{k-1}{2}, \frac{1+k}{2} ; 1 ; \frac{r^{2}}{a^{2}}\right) & \text { for } 0 \leq r \leq a \\
\frac{1-k}{2}\left(\frac{a}{r}\right){ }_{2}^{1+k} \mathrm{~F}_{1}\left(\frac{1+k}{2}, \frac{1+k}{2} ; 2 ; \frac{a^{2}}{r^{2}}\right) & \text { for } \quad r>a
\end{array} .\right.
$$

According to Eqs. (3) and (5), the shape of the indenter and the surface displacements of the power-law graded half-space both in the initial state and the steady wear state are depicted in Fig. 2 and 3. The gradient material in Fig. 2 is characterized by a negative exponent of elastic inhomogeneity $(k=-0.5)$, which maps a grading from a hard surface to a soft ground. In contrast, the displacements in Fig. 3 correspond to a power-law graded half-space with a soft surface and a hard ground $(k=0.5)$. 


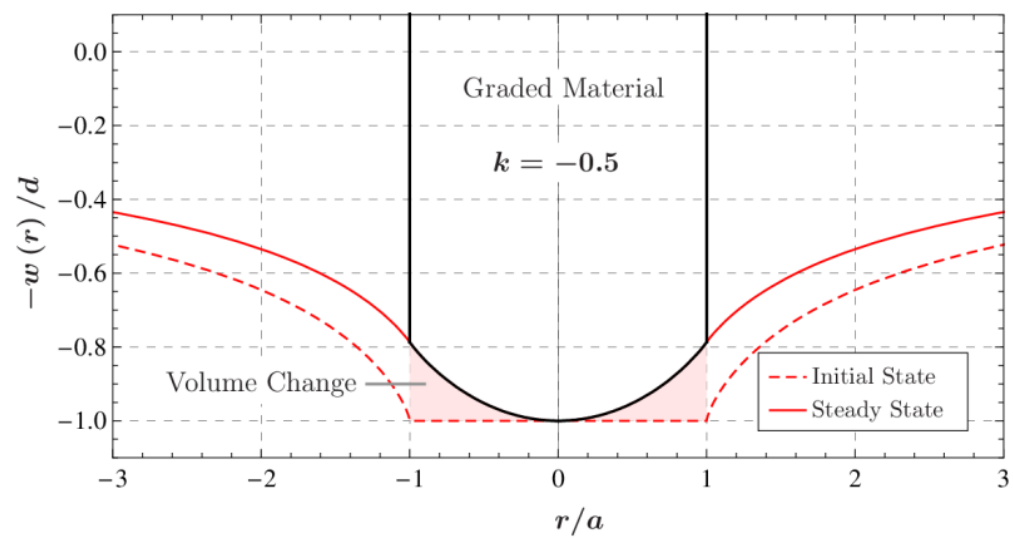

Fig. 2 Indenter profile and surface displacement of a power-law graded half-space with a negative power-law exponent $k=-0.5$ in the initial and the steady wear state

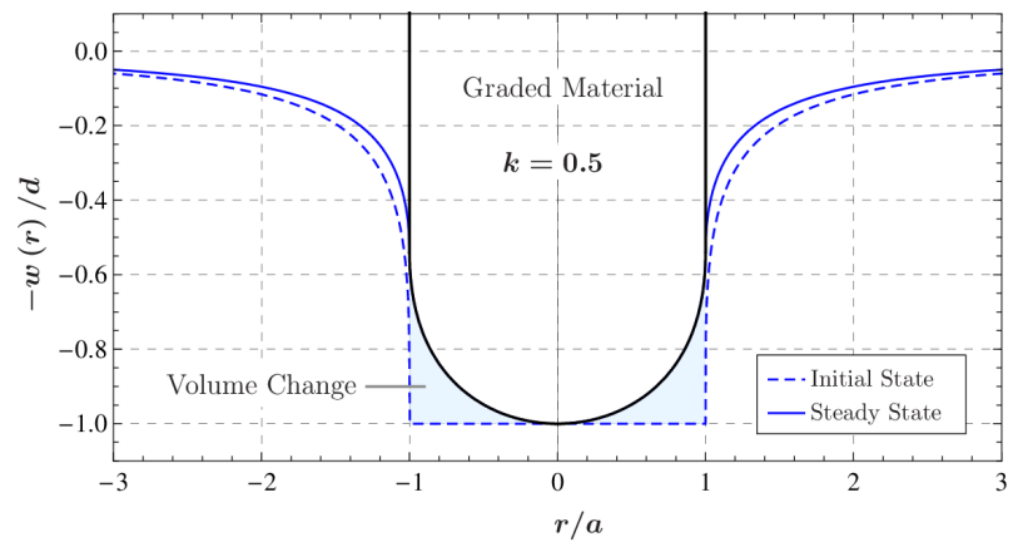

Fig. 3 Indenter profile and surface displacement of a power-law graded half-space with a positive power-law exponent $k=0.5$ in the initial and the steady wear state

A comparison of both indicates that the contact depth becomes larger and the surface displacements decrease with increasing the power-law exponent. Furthermore, the effective volume changes of the profile shape are highlighted by the shaded areas. Note that these volume changes do not represent absolute wear volumes. The effective volume change can be calculated as follows:

$$
\Delta V=\int_{0}^{a}\left(w_{0}(r)-w_{\infty}(r)\right) 2 \pi r \mathrm{~d} r
$$

Inserting Eqs. (3) and (5) into Eq. (6) yields

$$
\frac{\Delta V}{\pi a^{2} d}=1-{ }_{2} \mathrm{~F}_{1}\left(\frac{k-1}{2}, \frac{1+k}{2} ; 2 ; 1\right) .
$$


Fig. 4 illustrates the dependence of the normalized effective volume change on the exponent of elastic inhomogeneity. While it tends to zero at the edges of the exponent's domain, it reaches a maximum of $15.5 \%$ at a defined exponent of $k=0.169$. The absence of any change in the profile for $k=-1$ and $k=1$ is a consequence of the vanishing contact stiffness [29]. Only in the special case of a linear-inhomogeneous, incompressible halfspace there exists non-zero contact stiffness. This is the famous Gibson medium which responds to normal stresses on its surface like a Winkler foundation [30]. Therefore, once again, the initial shape of the profile does not change.

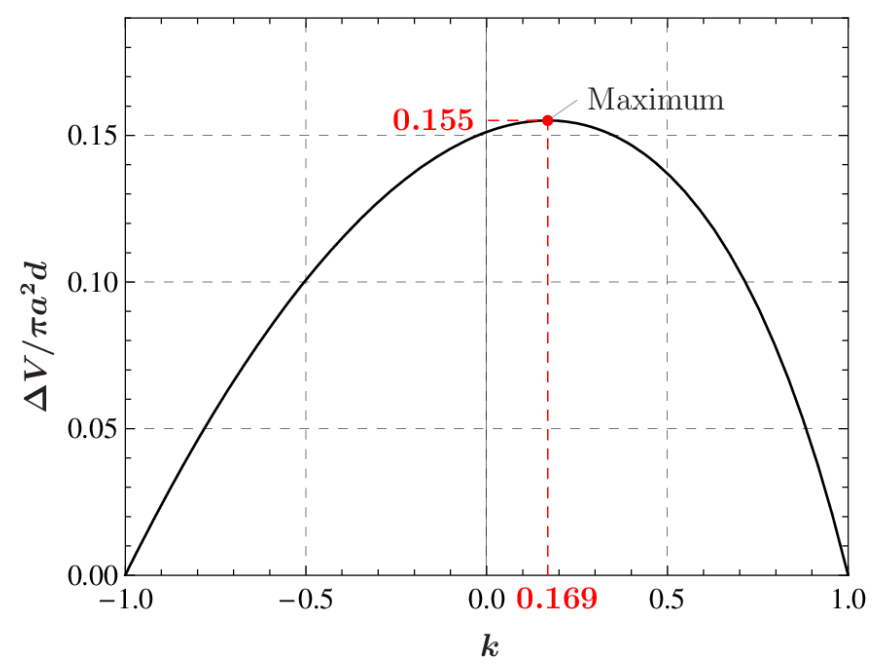

Fig. 4 Normalized effective volume change of the profile shape as a function of the exponent of the elastic inhomogeneity

Note that the wear process takes place under constant indentation depth. However, the shape variation leads to a redistribution of the contact pressure and thus a change of the normal force. Simple integration of the pressure distribution in the initial and the steady state over the contact area gives

$$
\begin{gathered}
\frac{F_{N, 0}}{2 E_{0} d a}=\frac{h_{N}(k, v)}{\left(1-v^{2}\right)(1+k)} \frac{a^{k}}{z_{0}^{k}}, \\
\frac{F_{N, \infty}}{2 E_{0} d a}=\frac{\pi h_{N}(k, v)(1-k)}{4\left(1-v^{2}\right) \cos \left(\frac{k \pi}{2}\right)} \frac{a^{k}}{z_{0}^{k}} .
\end{gathered}
$$

Fig. 5 demonstrates that the normal force generally decreases during the transition from the initial to the steady state, regardless of the choice of the characteristic depth. One could say that the system is trying to achieve an optimized state. The magnitudes of the normal forces according to Eqs. (8) and (9) strongly depend on both the exponent of elastic inhomogeneity $k$ and the characteristic depth $z_{0}$. The qualitative behavior of the normal force depending on these parameters can be taken from Fig. 5 and is compared in Table 1 with the behavior of the elastically homogeneous half-space. At small characteristic 
depths coupled with a positive power-law exponent or large characteristic depths coupled with a negative exponent, a larger normal force is required to produce the same indentation depth as for elastically homogeneous materials (exponents closer to -1 or 1 are excepted). In a very rough approximation one can compare the mentioned cases with the indentation of a layered half-space. Both a very thin and soft layer on a stiff substrate $\left(E_{S} \gg E_{0} \gg E_{L}\right)$ as well as a very thick and stiff layer $\left(E_{L} \gg E_{0} \gg E_{S}\right)$ on a soft substrate require a greater normal force for indentation. In a similar way, the tendencies associated with a decrease in the required normal force can be interpreted.

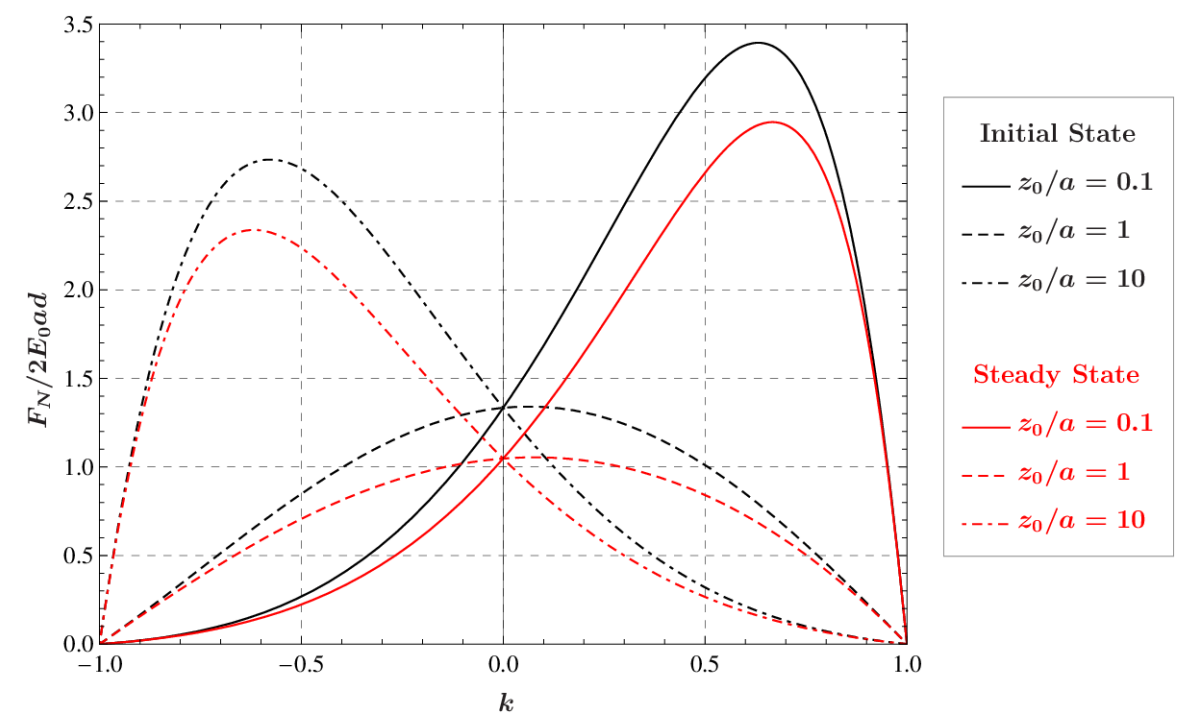

Fig. 5 Normal force in the initial and steady state as a function of the power-law grading exponent for different characteristic depths

Another interesting result is derived from the ratio of the normal forces given by Eqs. (9) and (8):

$$
\frac{F_{N, \infty}}{F_{N, 0}}=\frac{\pi(1-k)(1+k)}{4 \cos \left(\frac{k \pi}{2}\right)} .
$$

It is independent of the characteristic depth $z_{0}$ as well as an even function of exponent $k$ and confirms once again the fundamental decrease of the normal force.

Finally, let us briefly discuss the more practical boundary condition of gross slip under a constant normal force. Apart from a scaling factor, the relevant initial and steady state equations are the same as those given here. Under fixed normal force the indentation depth generally increases during the transition from the initial to the steady state. The ratio of the indentation depth in the final state to that in the initial state is given by the reverse of Eq. (10). Analogously, by rearranging of Eqs. (8) and (9), we obtain reverse curves to those shown in Fig. 5. In other words, very small values of the normal force under fixed penetration depth correspond to very high, and in the limit, even infinitely 
large values of the penetration depth under fixed load. This unphysical behavior is due to the choice of a power-law material gradient. Hence, we decided to prefer a study under fixed penetration depth.

Table 1 Tendencies of the change of normal force in comparison to an elastic homogeneous material

\begin{tabular}{ccc}
\hline & $z_{0}<a$ & $z_{0}>a$ \\
\hline$-1<k<0$ & $F_{\mathrm{N}} \downarrow$ & $F_{\mathrm{N}} \uparrow$ \\
$0<k<1$ & $F_{\mathrm{N}} \uparrow$ & $F_{\mathrm{N}} \downarrow$ \\
\hline
\end{tabular}

\section{Fretting WEAR ANALYSIS OF THE CONTACT BETWEEN A RigID PARABOLIC INDENTER AND A POWER-LAW GRADED HALF-SPACE}

We now come to the much more important partial slip contact problem between a rigid initially parabolic shaped indenter and a power-law graded half-space depicted in Fig. 6. The rigid indenter is pressed against the half-space by a fixed normal force $F_{N}$ and subsequently subjected by an oscillating tangential force $F_{x}$ whose magnitude does not exceed limiting value $\mu F_{\mathrm{N}}$. Once again, we suppose uncoupling of the normal and tangential contact as mentioned in the beginning of Chapt. 2 and assume that the indenter is rigid although it can wear. By contrast, wear of the power-law graded material should be neglected. At this point, it should be noted that all results are expected to remain valid even for opposite assumptions. Due to the change in the surface material properties of the worn gradient material, the redistribution of the pressure during the transient process will be different but the steady state should be unaffected by that. We consider very severe wear which means that wear proceeds until all partial slip is ceased.
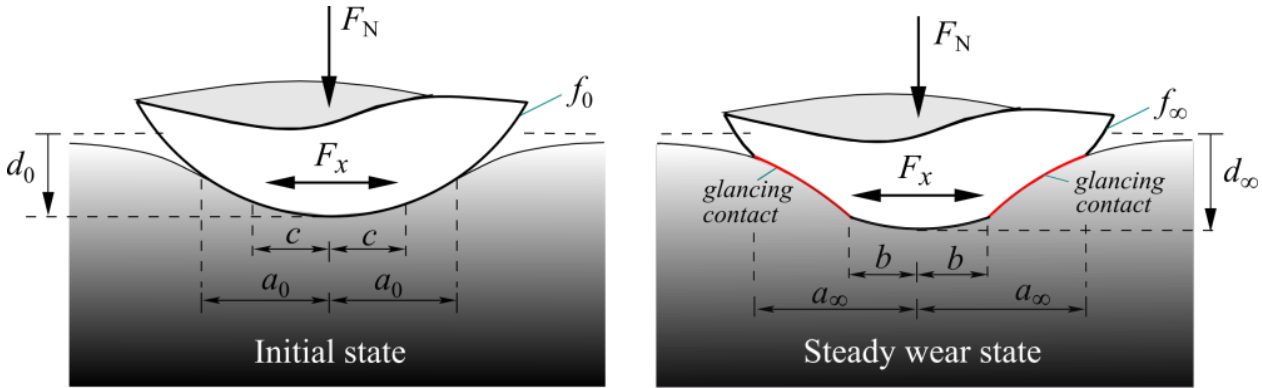

Fig. 6 Initial state (left) and steady state (right) of a partial slip contact problem between a rigid (initially) parabolic shaped indenter and a power-law graded half-space

In the initial state shown on the left in Fig. 6, the solution of the normal contact problem was derived by Giannakopoulos and Suresh [31]. The penetration depth, normal force, pressure distribution and normal surface displacement outside of the contact area are given by: 


$$
\begin{gathered}
d_{0}\left(a_{0}\right)=\frac{a_{0}^{2}}{(k+1) R}, \\
F_{N}\left(a_{0}\right)=\frac{4 h_{N}(k, v) E_{0} a_{0}^{k+3}}{\left(1-v^{2}\right) z_{0}^{k}(k+1)^{2}(k+3) R}, \\
p_{0}(r)=\frac{(k+3) F_{N}}{2 \pi a_{0}^{2}}\left[1-\left(\frac{r}{a_{0}}\right)^{2}\right]^{\frac{1+k}{2}}, \\
w_{0}(r)=\frac{a_{0}^{2} \cos \left(\frac{k \pi}{2}\right)}{(k+1) \pi R}\left[\mathrm{~B}\left(\frac{a_{0}^{2}}{r^{2}} ; \frac{1+k}{2}, \frac{1-k}{2}\right)-\frac{r^{2}}{a_{0}^{2}} \mathrm{~B}\left(\frac{a_{0}^{2}}{r^{2}} ; \frac{3+k}{2}, \frac{1-k}{2}\right)\right],
\end{gathered}
$$

where $\mathrm{B}(z ; x, y)$ represents the incomplete beta function according to

$$
\mathrm{B}(z ; x, y):=\int_{0}^{z} t^{x-1}(1-t)^{y-1} \mathrm{~d} t \quad \forall x, y \in \mathbb{R}^{+} .
$$

The solution of the tangential contact problem goes back to Heß [27]. The (minimum) stick radius $c$ as a function of (fixed) tangential force amplitude $F_{\mathrm{x}}$ is

$$
\frac{c}{a_{0}}=\left(1-\frac{F_{x}}{\mu F_{\mathrm{N}}}\right)^{\frac{1}{k+3}},
$$

where $a_{0}$ denotes the initial contact radius $[28,32]$. Herein, it was made use of the usual assumptions: The direction of the tangential stresses coincides with the direction of the applied tangential force. Moreover, the (small) slip component perpendicular to the applied tangential force is neglected.

In the steady state depicted on the right in Fig. 6 an inner part of the contact still remains in the state of stick so that in this region the final indenter profile coincides with the initial one. This stick region of radius $b \leq c$ is enclosed by an annular domain of outer radius $a_{\infty}$ in which the pressure becomes zero and the surfaces of the two bodies are in glancing contact. Thus, the steady contact state can be calculated by using the normal contact solution of a cylindrical punch of radius $b$ with a parabolic tip. The solution can be interpreted as a superposition of two parts:

I. The solution for the indentation by a parabolic indenter until contact radius $b$ is reached. Corresponding load $F_{1}$ is given by Eq. (12) if we replace $a$ with $b$.

II. A rigid body translation of the contact area of radius $b$ in vertical direction caused by an applied load $F_{\mathrm{N}}-F_{1}$ which corresponds to the indentation by a flat-ended cylindrical punch whose solution can be adopted from Eqs. (2) and (3). 
In this way we come to the following solution of the normal contact in the steady state:

$$
\begin{aligned}
p_{\infty}(r)= & \frac{(k+3) F_{1}(b)}{2 \pi b^{2}}\left[1-\left(\frac{r}{b}\right)^{2}\right]^{\frac{1+k}{2}}+\frac{(k+1)\left(F_{\mathrm{N}}-F_{1}(b)\right)}{2 \pi b^{2}}\left[1-\left(\frac{r}{b}\right)^{2}\right]^{\frac{k-1}{2}}, \\
w_{\infty}(r)= & \frac{b^{2} \cos \left(\frac{k \pi}{2}\right)}{(k+1) \pi R}\left[\mathrm{~B}\left(\frac{b^{2}}{r^{2}} ; \frac{1+k}{2}, \frac{1-k}{2}\right)-\frac{r^{2}}{b^{2}} \mathrm{~B}\left(\frac{b^{2}}{r^{2}} ; \frac{3+k}{2}, \frac{1-k}{2}\right)\right] \\
& +\frac{\cos \left(\frac{k \pi}{2}\right)\left(1-v^{2}\right)(1+k) z_{0}^{k}\left(F_{\mathrm{N}}-F_{1}(b)\right)}{2 \pi h_{N}(k, v) E_{0} b^{1+k}} \mathrm{~B}\left(\frac{b^{2}}{r^{2}} ; \frac{1+k}{2}, \frac{1-k}{2}\right) .
\end{aligned}
$$

The tangential contact in the steady state is characterized by a rigid body (tangential) displacement of the whole stick area. The corresponding distribution of tangential traction due to a tangential force $F_{\mathrm{x}}$ is [27]

$$
\tau_{\infty}(r)=\frac{(k+1) F_{x}}{2 \pi b^{2}}\left[1-\left(\frac{r}{b}\right)^{2}\right]^{\frac{k-1}{2}} .
$$

\subsection{Extent of the stick area in the steady state}

In the following, we will prove that the limiting stick radius $b$ in the steady state coincides with the minimum stick radius $c$ in the initial state. For this purpose, we study the distributions of pressure and tangential tractions at the vicinity of the edge of contact in the steady state. Let $r=b(1-\delta)$ with $\delta \ll<1$, then from Eqs. (17) and (19) we obtain the asymptotic expressions

$$
\begin{gathered}
p_{\infty}(\delta) \approx \frac{(k+1)\left(F_{\mathrm{N}}-F_{1}(b)\right)}{2 \pi b^{2}}(2 \delta)^{\frac{k-1}{2}}, \\
\tau_{\infty}(\delta) \approx \frac{(k+1) F_{x}}{2 \pi b^{2}}(2 \delta)^{\frac{k-1}{2}} .
\end{gathered}
$$

Both tend towards infinity like $\delta^{(\mathrm{k}-1) / 2}$. However, close to the edge of the contact (within the stick area) we expect tangential stresses which are only slightly smaller than the limiting value prescribed by Amontons law, i.e. $\tau_{\infty}(\delta)=\mu p_{\infty}(\delta)-\varepsilon$. Using Eq. (20) as well as Eq. (21) and taking the limit $\varepsilon \rightarrow 0$ yield

$$
\frac{F_{x}}{\mu F_{\mathrm{N}}}=\mu\left(1-\frac{F_{1}(b)}{F_{\mathrm{N}}}\right) .
$$

Remember that the normal force is kept constant during the transient wear process which is why we can substitute the normal force $F_{\mathrm{N}}$ on the right side of Eq. (22) by the expression given in Eq. (12). Since the force $F_{1}$ is also defined by Eq. (12) - we just have to replace radius $a_{0}$ by radius $b$, Eq. (22) gives 


$$
\frac{b}{a_{0}}=\left(1-\frac{F_{x}}{\mu F_{\mathrm{N}}}\right)^{\frac{1}{k+3}} \text {. }
$$

A comparison with Eq. (16) proves that the radius of the stick area in the steady state is indeed equal to the radius of the minimum stick area in the initial state.

\subsection{Limiting shape of the profile in the steady state}

The shape $f_{\infty}$ of the steady state profile in the worn area is determined by the displacement of the free half-space surface due to the indentation by a cylindrical punch of radius $c$ with a parabolic tip. Taking into account $b=c$ as well as Eq. (12), Eq. (18) results in

$$
\begin{aligned}
\frac{w_{\infty}(\tilde{r})}{a_{0}^{2} / R}= & \frac{\cos \left(\frac{k \pi}{2}\right) \tilde{c}^{2}}{\pi(k+3)}\left(1+\frac{2}{k+1} \frac{1}{\tilde{c}^{k+3}}\right) \mathrm{B}\left(\frac{\tilde{c}^{2}}{\tilde{r}^{2}} ; \frac{1+k}{2}, \frac{1-k}{2}\right), \\
& -\frac{\cos \left(\frac{k \pi}{2}\right) \tilde{r}^{2}}{\pi(k+1)} \mathrm{B}\left(\frac{\tilde{c}^{2}}{\tilde{r}^{2}} ; \frac{3+k}{2}, \frac{1-k}{2}\right)
\end{aligned}
$$

where we have introduced $\tilde{c}=c / a_{0}$ and $\tilde{r}=r / a_{0}$.

As can be seen in Fig. 6 on the right, the steady state wear profile is defined as follows:

$$
f_{\infty}(r)=\left\{\begin{array}{l}
f_{0}(r) \text { for } 0 \leq r<c \vee r>a_{\infty} \\
d_{\infty}-w_{\infty}(r) \text { for } c \leq r \leq a_{\infty}
\end{array} .\right.
$$

However, the outer radius of worn region $a_{\infty}$ is still unknown and must be determined from the condition that the initial profile and the steady state profile coincide at this point:

$$
f_{\infty}\left(a_{\infty}\right)=f_{0}\left(a_{\infty}\right) \Rightarrow f_{0}\left(a_{\infty}\right)=f_{0}(c)+w_{\infty}(c)-w_{\infty}\left(a_{\infty}\right) .
$$

Normalized by the contact radius in the initial state, Fig. 7 shows the limiting radii of the worn annular region in the steady state as a function of the normalized amplitude of the applied tangential force according to Eqs. (16) and (26). Curves are plotted for both a homogeneous material and two power-law graded materials characterized by exponents $k=-0.5$ and $k=0.5$. It is obvious that the larger the exponent of elastic inhomogeneity the larger the stick radius $c$ and the smaller the radius of the maximum extent of worn region $a_{\infty}$. However, this statement should be treated with caution since the radii have been normalized to contact radius $a_{0}$ in the initial state and the latter depends strongly on both the exponent of elastic inhomogeneity and the characteristic depth. To be able to compare absolute values, we would have to presuppose power-law graded materials, which exhibit the same contact area in the initial contact state if subjected by the same fixed normal force. Such conditions could be of interest in designing an electrical contact, which require both a fixed normal force and a fixed contact area to transmit a certain current (the change in conductivity due to the material gradient is disregarded). 


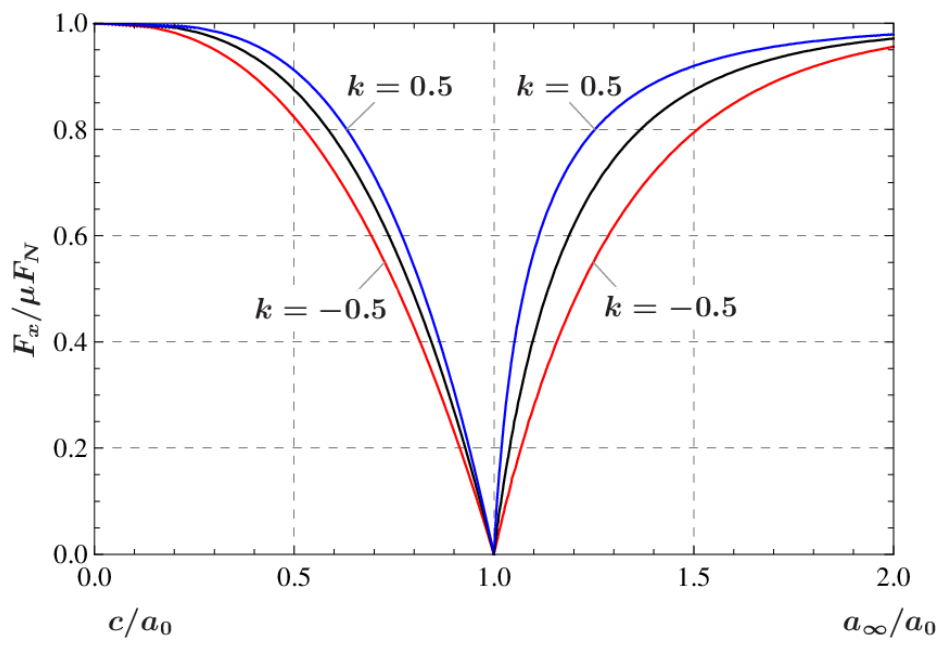

Fig. 7 Inner radius $c$ and outer radius $a_{\infty}$ of the annular worn region in the steady state versus aspect traction ratio $F_{\mathrm{x}} / \mu F_{N}$

Three sample materials that meet these conditions are characterized by the following elastic material gradients:

$$
\text { 1. } E(z)=E_{0} \quad 2 . E(z)=0.88 E_{0} \sqrt{a_{0} / z} \quad 3 . E(z)=2.06 E_{0} \sqrt{z / a_{0}} .
$$

That the entire worn area decreases with increasing exponent of elastic inhomogeneity, is also evident in Fig. 8. In this Figure, the final shapes of the wear profile for 3 different materials are compared: at the top an inhomogeneous material with $k=-0.5$, which is characterized by a hard surface and a soft ground; in the middle an elastically homogeneous material and at the bottom an inhomogeneous material with $k=0.5$, which maps a half-space characterized by a soft surface and a hard ground. In addition to the initial profile of the rigid indenter, its wear profiles are shown for three different amplitudes of the tangential force. It need not be mentioned that in all cases the wear volume grows with increasing amplitude of the tangential force. But Fig. 8 also indicates that in comparison to the homogeneous case, the wear volume is greater for negative exponents and smaller for positive ones. In other words, for the three tangential force amplitudes considered, the wear in the case of gradient materials with a soft surface and a hard ground is smaller than that for those with a hard surface and a soft ground. However, that this statement is not universally valid is supported by Fig. 9, in which the wear volume is plotted as a function of the amplitude of the tangential force for different exponents of elastic inhomogeneity. The worn volume was determined according to

$$
\Delta V=\int_{c}^{a_{\infty}}\left(f_{\infty}(r)-f_{0}(r)\right) 2 \pi r \mathrm{~d} r=\int_{c}^{a_{\infty}}\left(w_{\infty}(0)-w_{\infty}(r)-f_{0}(r)\right) 2 \pi r \mathrm{~d} r .
$$



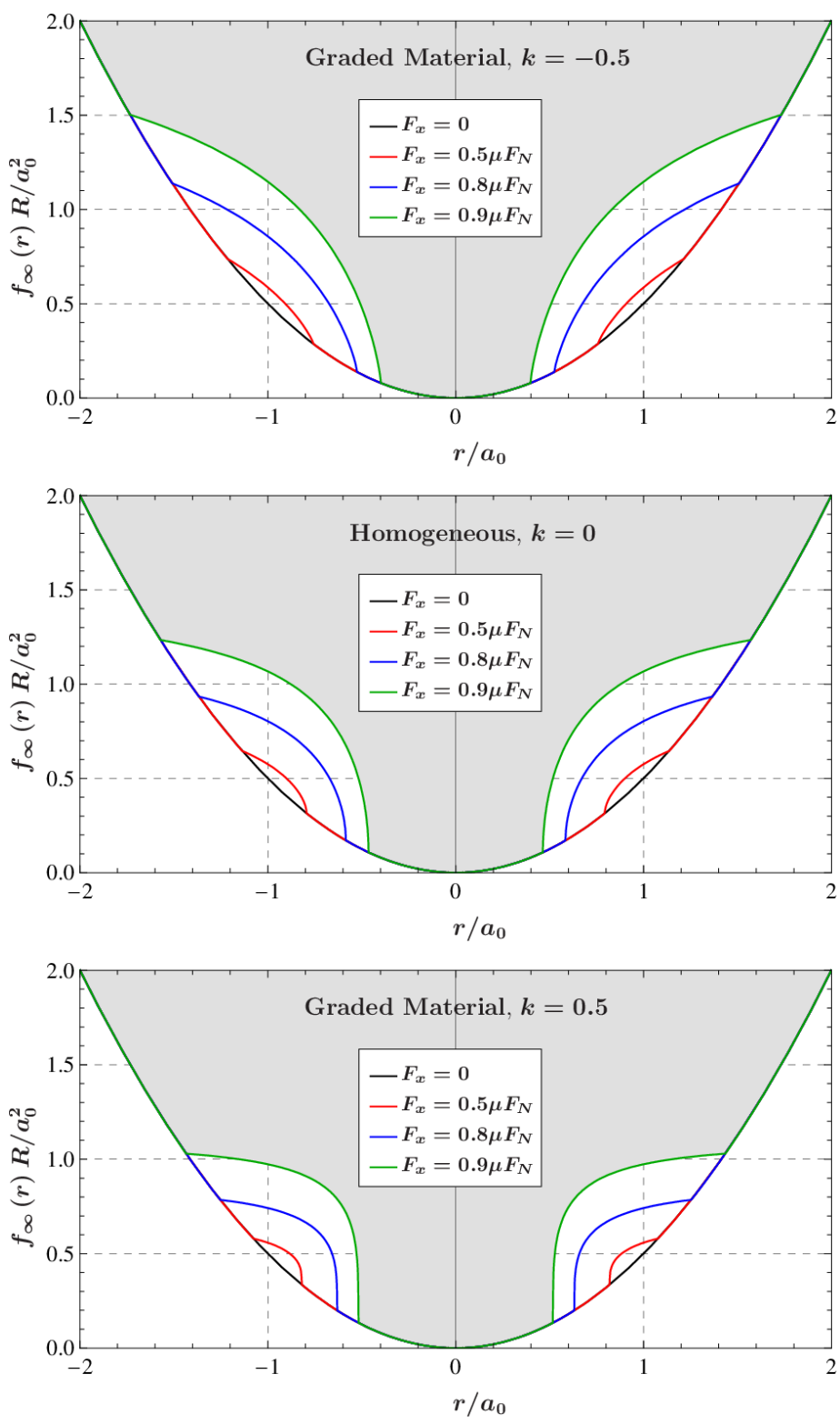

Fig. 8 Limiting shapes of the profile for three different tangential force amplitudes and three different exponents of elastic inhomogeneity: $k=-0.5$ (top), $k=0$ (middle) and $k=0.5$ (bottom)

It is clearly visible that amplitudes less than about $20 \%$ of the limiting value $\mu F_{\mathrm{N}}$ cause more wear if the materials show a gradient ranged from a soft surface to a hard 
ground. This is a significant result which can be important for optimized material selection of many technical systems. Numerous force-fitted connections are characterized by a very high normal force to realize a preferably large stick region. However, it is wellknown that even the smallest vibrations perpendicular to the normal load result in a small annulus of slip which inevitably causes energy dissipation and wear. According to our results, in the mentioned range of small tangential force amplitudes, the use of a powerlaw gradient material with a negative exponent would reduce wear. Thus, a material gradient is needed that ranges from a hard surface to a soft ground. In contrast, an operating area of high tangential force amplitudes requires a power-law graded material with a positive exponent to minimize wear. The tendencies in the behavior of the wear volume depending on the material gradient can be intuited from Fig. 8. It reveals that the amount of curvature of the worn area increases significantly with increasing exponent, whereas the worn area itself decreases. For very small slip annuli, the influence of the curvature on the wear volume seems to predominate.

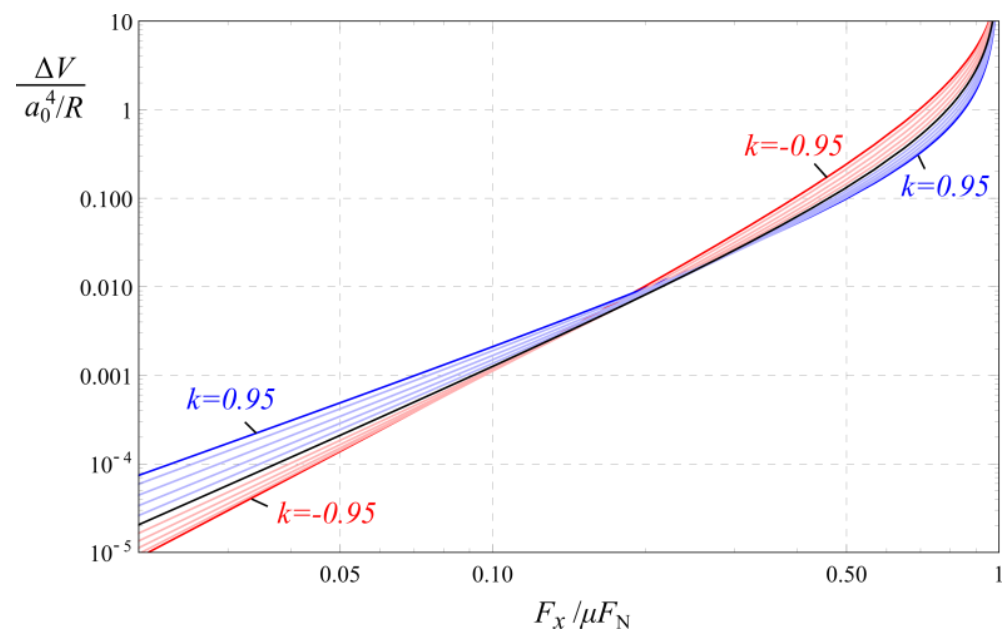

Fig. 9 Normalized representation of the wear volume as a function of the tangential force amplitude for different exponents of elastic inhomogeneity; red curves indicate a negative exponent, blue curves a positive exponent

In Sec. 3.1, we have already examined the normal and tangential stresses near the edge of the stick area in the fully worn steady state. In Fig. 9 the pressure distribution in the fully worn steady state for three different tangential force amplitudes and three different exponents of elastic inhomogeneity are displayed $-k=-0.5$ at the top, $k=0$ in the middle and $k=0.5$ at the bottom. Once again it is clearly visible that the stick radius and thus the area for transmitting the normal force grow with increasing exponent of elastic inhomogeneity. This is tantamount with a decrease of the mean pressure. Furthermore, the stress singularity becomes milder with increasing exponent $k$. The same applies to the singularity of the tangential stresses according to Eq. (21). The stress field resembles the near-tip stress field of a mixed-mode II/III crack, whose weighting depends on the surrounding position on the edge of contact. On the $x$-axis it corresponds to a pure mode 
II loading, whereas on the $y$-axis to a pure mode III loading. The influence of the exponent of elastic inhomogeneity on the stress singularity can be profitably used to suppress plastic yielding and crack nucleation at the edge of contact.
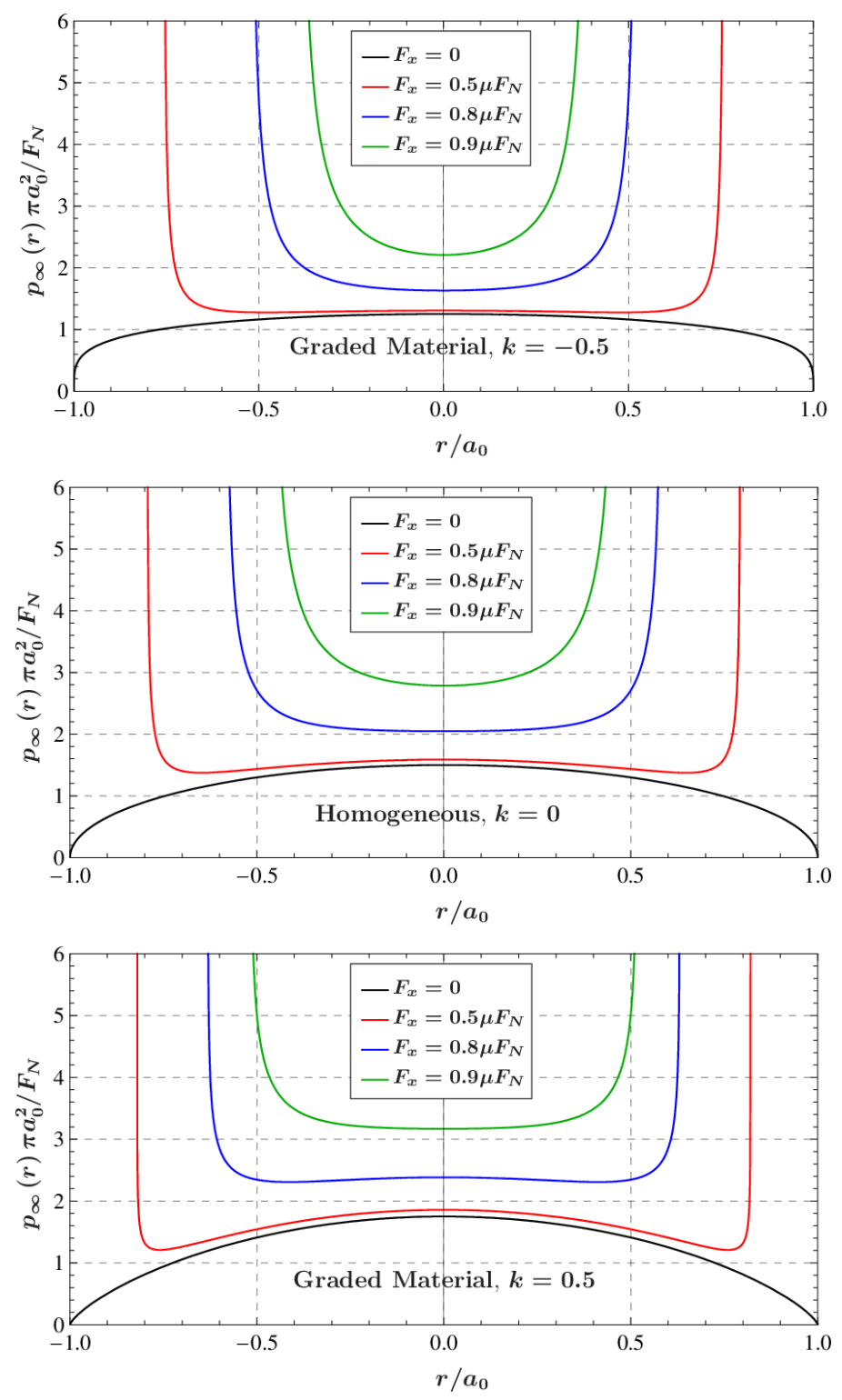

Fig. 9 Pressure distribution in the fully worn steady state for three different tangential force amplitudes and three different exponents of elastic inhomogeneity: $k=-0.5$ (top), $k=0$ (middle) and $k=0.5$ (bottom) 


\section{CONCLUSIONS AND DISCUSSION}

We have investigated the steady wear state for two types of frictional contact problems between a rigid indenter and a power-law graded material. First, we considered a gross slip between a rigid, cylindrical punch with an initially flat circular base and a power-law graded half-space. Under fixed penetration depth and the strong assumption that merely the indenter can wear, the solutions for the initial contact state as well as the steady wear state are given in terms of pressure distribution and normal surface displacements. Moreover, the steady wear shape of the profile and the effective volume change were determined. We have found that the effective volume change becomes a maximum of $15.5 \%$ at a defined exponent of $k=0.169$. For power-law graded materials characterized by either small characteristic depths coupled with a positive power-law exponent or large characteristic depths coupled with a negative exponent, a larger normal force is required to produce the same indentation depth as for elastically homogeneous material.

Second, we analyzed fretting wear under load-controlled conditions for the contact between a rigid initially parabolic indenter and a power-law graded half-space. Again, complete solutions are given in terms of stresses and normal surface displacements for both the initial and the steady state. Following previous studies for homogeneous materials, we prove that the size of the stick zone does not change during the transition from the initial to the steady state. As mentioned above, the power-law graded half-space is assumed to be resistant to wear but the rigid indenter should wear. The dependence of limiting profile shapes $f_{\infty}$ on the exponent of elastic inhomogeneity as well as on the tangential force has been completely determined. One interesting result is that the amount of curvature of the worn area increases significantly with increasing exponent. But probably the most significant result of our analyses is the finding that opposing material gradients for small and large tangential force amplitudes are necessary to minimize the wear. Whereas for large amplitudes a gradient ranged from a soft surface to a hard ground is beneficial, small amplitudes require a reverse gradient characterized by a hard surface and a soft ground. Although it is frequently stated in the literature that power-law graded materials reflect the qualitative behavior of real gradient materials quite well, appropriate analytical and experimental investigations should be made to ensure this result. The same applies to the influence of the milder stress singularity on fretting fatigue crack initiation.

In addition, we would like to emphasize that all our studies are restricted to purely power-law graded elastic material behavior. However, in practice, stress singularities are avoided by plastic deformation of the material and this in turn strongly influence the wear state. By using a finite-element model and considering elastic-plastic material behavior, $\mathrm{Hu}$ et al. [33] found that the stick-slip boundary can move steadily into the stick region and thus wear continues indefinitely. Thus, future investigations on wear of functionally graded materials should consider the elastic-plastic regime.

Acknowledgement: The author would like to thank Dr. R. Pohrt and Prof. V. L. Popov for many valuable discussions. 


\section{APPENDIX}

The function $h_{N}(k, v)$ in Eq. (2) is defined by (see [32])

$$
h_{N}(k, v)=\frac{2(1+k) \cos \left(\frac{k \pi}{2}\right) \Gamma\left(1+\frac{k}{2}\right)}{\sqrt{\pi} C(k, v) \beta(k, v) \sin \left(\frac{\beta(k, v) \pi}{2}\right) \Gamma\left(\frac{1+k}{2}\right)},
$$

with

$$
C(k, v)=\frac{2^{1+k} \Gamma\left(\frac{3+k+\beta(k, v)}{2}\right) \Gamma\left(\frac{3+k-\beta(k, v)}{2}\right)}{\pi \Gamma(2+k)}
$$

and

$$
\beta(k, v)=\sqrt{(1+k)\left(1-\frac{k v}{1-v}\right)} .
$$

\section{REFERENCES}

1. Kim, D.G., Lee, Y.Z., 2001, Experimental investigation on sliding and fretting wear of steam generator tube materials, Wear, 250(1-12), pp. 673-680.

2. Rajasekaran, R., Nowell, D., 2006, Fretting fatigue in dovetail blade roots: Experiment and analysis, Tribology international, 39(10), pp. 1277-1285.

3. Dyrkacz, R.M., Brandt, J.M., Ojo, O.A., Turgeon, T.R., Wyss, U.P., 2013, The influence of head size on corrosion and fretting behaviour at the head-neck interface of artificial hip joints, The Journal of arthroplasty, 28(6), pp. 1036-1040.

4. Fouvry, S., Jedrzejczyk, P., Chalandon, P., 2011, Introduction of an exponential formulation to quantify the electrical endurance of micro-contacts enduring fretting wear: Application to Sn, Ag and Au coatings, Wear, 271(9-10), pp. 1524-1534.

5. Martínez, J.C., Useche, L.V.V., Wahab, M.A., 2017, Numerical prediction of fretting fatigue crack trajectory in a railway axle using XFEM, International Journal of Fatigue, 100, pp. 32-49.

6. Hills, D.A., Sackfield, A., Paynter, R.J.H., 2009, Simulation of fretting wear in halfplane geometries: part 1the solution for long term wear, Journal of Tribology, 131(3), 031401.

7. Dini, D., Sackfield, A., Hills, D.A., 2008, An axi-symmetric Hertzian Contact subject to cyclic shear and severe wear, Wear, 265(11-12), pp. 1918-1922.

8. Popov, V. L., 2014, Analytic solution for the limiting shape of profiles due to fretting wear, Scientific reports, 4, 3749.

9. Argatov, I.I., Chai, Y.S., 2018, Limiting shape of profiles in fretting wear, Tribology International, 125, pp. 95-99.

10. Ciavarella, M., Hills, D.A., 1999, Brief note: Some observations on oscillating tangential forces and wear in general plane contacts, European Journal of Mechanics-A/Solids, 18(3), pp. 491-497.

11. Archard, J., 1953, Contact and rubbing of flat surfaces, Journal of applied physics, 24(8), pp. 981-988.

12. Ding, J., Leen, S.B., McColl, I.R., 2004, The effect of slip regime on fretting wear-induced stress evolution, International journal of fatigue, 26(5), pp. 521-531.

13. Goryacheva, I.G., Rajeev, P.T., Farris, T.N., 2001, Wear in partial slip contact, Journal of Tribology, 123(4), pp. 848-856.

14. Nowell, D., 2010, Simulation of fretting wear in half-plane geometries-Part II: analysis of the transient wear problem using quadratic programming, Journal of Tribology, 132(2), 021402. 
15. Popov, V.L., Heß, M., 2015, Method of dimensionality reduction in contact mechanics and friction, Springer Berlin Heidelberg, ISBN: 978-3-642-53875-9.

16. Dimaki, A.V., Dmitriev, A.I., Menga, N., Papangelo, A., Ciavarella, M., Popov, V.L., 2016, Fast high-resolution simulation of the gross slip wear of axially symmetric contacts, Tribology Transactions, 59(1), pp. 189-194.

17. Dimaki, A.V., Dmitriev, A.I., Chai, Y.S., Popov, V.L., 2014, Rapid simulation procedure for fretting wear on the basis of the method of dimensionality reduction, International Journal of Solids and Structures, 51(25-26), pp. 4215-4220.

18. Li, Q., Filippov, A. E., Dimaki, A. V., Chai, Y.S., Popov, V.L., 2014, Simplified simulation of fretting wear using the method of dimensionality reduction, Physical Mesomechanics, 17(3), pp. 236-241.

19. Dmitriev, A. I., Voll, L. B., Psakhie, S. G., Popov, V. L., 2016, Universal limiting shape of worn profile under multiple-mode fretting conditions: theory and experimental evidence, Scientific reports, 6, 23231.

20. Suresh, S., 2001, Graded materials for resistance to contact deformation and damage, Science, 292(5526), pp. 2447-2451

21. Jitcharoen, J., Padture, N.P., Giannakopoulos, A. E., Suresh, S., 1998, Hertzian- crack suppression in ceramics with elastic- modulus- graded surfaces, Journal of the American Ceramic Society, 81(9), pp. 2301-2308

22. Ke, L.L., Wang, Y.S., 2010, Fretting contact of two dissimilar elastic bodies with functionally graded coatings, Mechanics of Advanced Materials and Structures, 17(6), pp. 433-447.

23. Guler, M. A., Ozturk, M., Kucuksucu, A., 2016, The frictional contact problem of a rigid stamp sliding over a graded medium, Key Engineering Materials, 681, pp. 155-174.

24. Wang, Z., Yu, C., Wang, Q., 2015, An efficient method for solving three-dimensional fretting contact problems involving multilayered or functionally graded materials, International Journal of Solids and Structures, 66, pp. 46-61.

25. Booker, J.R., Balaam, N.P., Davis, E.H., 1985, The behaviour of an elastic non-homogeneous half-space. Part II-circular and strip footings, International journal for numerical and analytical methods in geomechanics, 9(4), pp. 369-381.

26. Jin, F., Guo, X., Zhang, W., 2013, A unified treatment of axisymmetric adhesive contact on a power-law graded elastic half-space, Journal of Applied Mechanics, 80(6), 061024.

27. Heß, M., 2016, Normal, tangential and adhesive contacts between power-law graded materials. Presentation at the Workshop on Tribology and Contact Mechanics in Biological and Medical Applications, TU-Berlin, 14.-17. Nov. 2016

28. Heß, M., Popov, V.L., 2016, Method of dimensionality reduction in contact mechanics and friction: a user's handbook. II. Power-law graded materials, Facta Universitatis, Series: Mechanical Engineering, 14(3), pp. 251-268.

29. Heß, M., 2016, A simple method for solving adhesive and non-adhesive axisymmetric contact problems of elastically graded materials, International Journal of Engineering Science, 104, pp. 20-33.

30. Gibson, R. E., 1967, Some results concerning displacements and stresses in a non-homogeneous elastic halfspace. Geotechnique, 17(1), pp. 58-67.

31. Giannakopoulos, A.E., Suresh, S., 1997, Indentation of solids with gradients in elastic properties: Part II. Axisymmetric indentors, International Journal of Solids and Structures, 34(19), pp. 2393-2428.

32. Popov, V.L., Heß, M., Willert, E., 2018, Handbuch der Kontaktmechanik: Exakte Lösungen axialsymmetrischer Kontaktprobleme, Springer-Verlag, ISBN: 978-3-662-53010-8.

33. Hu, Z., Lu, W., Thouless, M.D., Barber, J.R., 2016, Effect of plastic deformation on the evolution of wear and local stress fields in fretting, International Journal of Solids and Structures, 82, pp. 1-8. 